\title{
Adhesion of bio-functionalized ultrasound microbubbles to endothelial cells by targeting to vascular cell adhesion molecule-I under shear flow
}

\author{
This article was published in the following Dove Press journal: \\ International Journal of Nanomedicine \\ 20 September 2011 \\ Number of times this article has been viewed
}

\section{Hong Yang \\ Xiaoyan Xiong \\ Lie Zhang \\ Chunhui Wu \\ Yiyao Liu}

Department of Biophysics, School of Life Science and Technology, University of Electronic Science and Technology of China, Chengdu, Sichuan, People's Republic of China
Correspondence: Yiyao Liu

Department of Biophysics, School

of Life Science and Technology,

University of Electronic Science and

Technology of China, Chengdu 610054,

Sichuan, People's Republic of China

Tel +862883203353

Fax +862883208238

Email liuyiyao@hotmail.com

\begin{abstract}
The expression of certain endothelial cell adhesion molecules is increased during endothelial dysfunction or inflammatory activation. This has led to the concept of using microbubbles for targeted molecular imaging or drug delivery. In this approach, microbubbles with a specific ligand to receptors expressed at the site of specific diseases are constructed. The present study aimed to engineer a novel type of bio-functionalized microbubbles (vascular cell adhesion molecule 1 [VCAM-1]-targeted microbubbles), and determine whether VCAM-1-targeted microbubbles exhibit specific adhesion to lipopolysaccharide (LPS)-activated endothelial cells. Our data showed that VCAM-1 expression was significantly upregulated in both LPS-activated endothelial cells in vitro and endothelium in a rat atherosclerosis model in vivo. Targeted microbubbles were designed by conjugating anti-VCAM-1 monoclonal antibodies to the shell of microbubbles using biotin-avidin bridging chemistry methods. Microbubble adhesion to endothelial cells was assessed in a flow chamber at two shear stress conditions (6.3 and 10.4 dynes $/ \mathrm{cm}^{2}$ ). Our data showed that microbubble adhesion depends on both the surface antiVCAM-1 antibody densities and the exposed shear stresses. Adhesion of VCAM-1-targeted microbubbles onto LPS-activated endothelial cells increased with the surface antibody densities, and decreased with the exposed shear stresses. These findings showed that the specific ligandcarrying microbubbles have considerable potential in targeted ultrasound molecular imaging or ultrasound-assisted drug/gene delivery applications.
\end{abstract}

Keywords: targeted microbubbles, VCAM-1, adhesion, HUVEC-CS, shear flow

\section{Introduction}

Ultrasound contrast agents composed of gas-filled microbubbles with diameters generally ranging from 2 to $8 \mu \mathrm{m}$ have been in clinical use for many years. ${ }^{1,2}$ These agents can improve accuracy and imaging contrast for targeted sites of interest during ultrasound imaging. In the last 10 years, several site-targeted microbubbles have been developed, and have been used for molecular and cellular imaging in vitro and in vivo, ${ }^{3,4}$ as well as for ultrasound-assisted drug/gene delivery and triggered release..$^{5-7}$

The expression of endothelial cell adhesion molecules known to play an important role in leukocyte recruitment (ie, VCAM-1, E-selectin, P-selectin, and ICAM-1), are often increased at the sites of pathological inflammation. ${ }^{8}$ This observation has led to a strong interest in the development of targeted delivery strategies that exploit the increased expression of endothelial cell adhesion molecules to achieve selective delivery to the sites of interest. VCAM-1, expressed by inflammatory endothelial cells, participates in 
leukocyte rolling and adhesion primarily by interacting with its counterligand $\alpha_{4} \beta_{1}$ on monocytes and lymphocytes. VCAM-1 on the vessel endothelial surface also plays an important role in atherosclerosis development. Atherosclerosis is regarded as a chronic inflammatory disease, and endothelial dysfunction is one of the key events in the pathogenesis of atherosclerosis. Previous studies have been reported in which overexpression of VCAM-1 and/or other cell adhesion molecules has been associated with endothelial cell dysfunction and atherosclerosis progression. ${ }^{9,10}$ This suggests that VCAM-1 is an ideal target for microbubble targeted delivery.

We hypothesized that targeted microbubbles could be prepared by coupling the ligands of endothelial cell adhesion molecules onto the microbubble surface. The bio-functionalized microbubbles would then bind to the specific endothelial cell adhesion molecules whose expressions were upregulated on the endothelial cell surfaces, and be retained in the inflamed endothelium. It was necessary to profile the bio-functionalized microbubble adhesion in vitro under flow conditions. A parallel plate flow chamber system has been widely used to study the adhesion and interaction of neutrophils, platelet, and cancer cells with endothelial cells under flow conditions. ${ }^{11-13}$

In this study, a parallel flow chamber system was used to investigate the adhesion of VCAM-1-targeted microbubbles to an endothelial cell monolayer under controlled shear conditions. The process of adhesion was also examined, which will be helpful in the prediction of in vivo adhesion behavior of targeted microbubbles, as well as their future clinical applications.

\section{Materials and methods Materials}

Cell culture medium RPMI 1640 and newborn calf serum (NCS) were purchased from Gibco (Grand Island, NY). Lipopolysaccharide (LPS) (from Escherichia coli 055:B5), trypsin, HAT (hypoxanthine, aminopterin, and thymidine) supplement, polyoxyethylene-40 (PEG-40) stearate, penicillin and streptomycin were obtained from Sigma-Aldrich (St Louis, MO). 2-[4-(2-Hydroxyethyl)-1-piperazine]ethanesulfonic acid (HEPES) was supplied by Amresco (Cleveland, OH). Distearoylphosphatidylcholine (DSPC) and distearoylphosphatidylethano- lamine-biotin (DSPE-biotin) were from Avanti Polar Lipids (Alabaster, AL). Biotinylated anti-VCAM-1 monoclonal antibody, streptavidin, and phycoerythrin-labeled streptavidin (PE-streptavidin) were obtained from BioLegend (San Diego, CA). Rabbit anti-human VCAM-1 antibody and goat anti-rabbit secondary antibody were from ZSGB-BIO (Beijing, China). All other chemicals used were of analytic grade without further purification, if not stated otherwise.

\section{Preparation of bio-functionalized ultrasound microbubbles}

Microbubbles with monoclonal antibodies against VCAM-1 conjugated to their surfaces were constructed using a multistep biotin-avidin bridging chemistry method described previously with some modifications. ${ }^{3,14}$ Briefly, the DSPC and DSPE-biotin were dispersed along with the surfactant PEG-40 stearate in chloroform solvent. The solvent was evaporated at $58^{\circ} \mathrm{C}$, and replaced with a phosphate buffer saline (PBS). The mixture was sonicated for 30 seconds in the presence of perfluoropropane $\left(\mathrm{C}_{3} \mathrm{~F}_{8}\right)$ gas. The sonication dispersed the perfluoropropane gas in the PBS solution during the microbubble formation. Static flotation was used to remove unincorporated lipids from microbubbles by triple washing with PBS. The biotinylated microbubbles were incubated with PE-streptavidin in PBS for 60 minutes at $4^{\circ} \mathrm{C}$, and the unbound PE-streptavidin was removed by static flotation as described above. The PE-streptavidin coated biotinylated microbubbles were then incubated with biotinylated antiVCAM-1 monoclonal antibody or isotype control biotinylated antibody $(\operatorname{IgG})$ for 60 minutes at $4^{\circ} \mathrm{C}$, and the unbound antibody was removed by static flotation, using triple washing with PBS. Targeted microbubbles were thus prepared with biotinylated anti-VCAM-1 monoclonal antibody coupled to the phospholipid monolayer of the microbubble shell through a biotin-streptavidin bridge (Figure 1).

\section{Biophysical characterization of microbubbles}

To observe the morphology of the prepared microbubbles, 3,3'dioctadecyloxacarbocyanine perchlorate (DiO) (Molecular

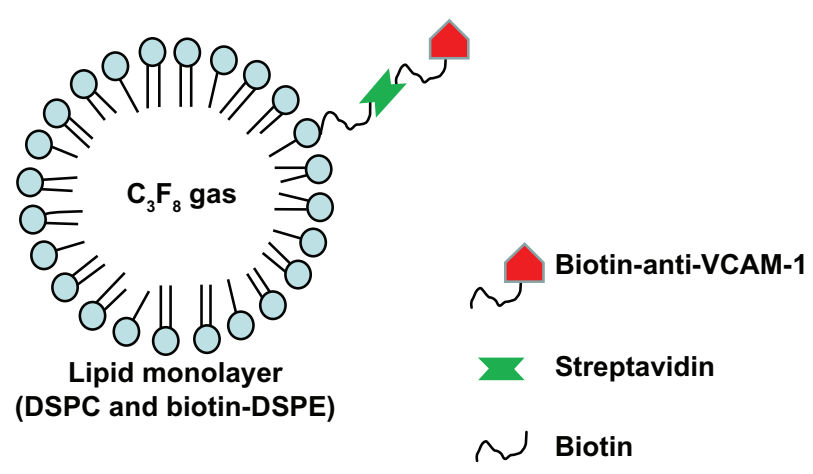

Figure I Schematic illustration for surface bio-functionalization to form VCAM-I targeted microbubbles through a biotin-streptavidin bridging chemistry method. Abbreviation: VCAM-I, vascular cell adhesion molecule I. 
Probes, Eugene, OR), a cell membrane green fluorescent probe, was used to label the shell of microbubbles. Microbubble suspension $(500 \mu \mathrm{L})$ was incubated with $1 \mathrm{~mL}$ DiO solution $(10 \mu \mathrm{M})$ for $20-30$ minutes, followed by triple washing in PBS to remove the excess DiO. The labeled microbubbles were detected under an inverted fluorescence microscope (TE-2000U; Nikon, Tokyo, Japan). Five hundred microliters of microbubble solution was diluted into $1 \mathrm{~mL}$ deionized water, and then the size distribution of microbubbles was determined using a Zetasizer Nano ZS (Malvern Instruments, Malvern, UK) according to the manufacturer's operating manual.

To quantify the anti-VCAM-1 antibody coverage, microbubbles were linked to PE-labeled streptavidin. Various concentrations of PE-streptavidin ranging from $0.02 \mu \mathrm{g}$ to $0.6 \mu \mathrm{g} / 100 \mu \mathrm{L}$ microbubble suspension (approximately $\left.1 \times 10^{8}\right)$ in PBS were used, and incubated for 60 minutes at room temperature. The unbound PE-streptavidin was removed by static flotation with triple washing in PBS, and the fluorescence intensity was detected by spectrofluorophotometry (RF-7000; Shimadzu, Kyoto, Japan). It was recognized that one streptavidin will bind one biotinylated anti-VCAM-1 monoclonal antibody, and the antibody coverage percentage (ie, antibody surface density) could be evaluated using a standard curve of PE-streptavidin fluorescence intensity under various PE-streptavidin concentrations.

\section{Cell culture and activation}

HUVEC-CS cells, a subline of human umbilical vein endothelial cells (HUVEC), ${ }^{15}$ were obtained from the American Type Culture Collection (Manassas, VA), and cultured in a medium consisting of cell culture medium RPMI1640 supplemented with $10 \%$ NCS and $1 \%$ penicillin/streptomycin. Cells were maintained in an incubator at $37^{\circ} \mathrm{C}$ with $95 \%$ humidity and $5 \% \mathrm{CO}_{2}$. HUVEC-CS cells were subcultured after trypsinization $(0.025 \%$ trypsin, $0.5 \mathrm{mM}$ EDTA, $1 \mathrm{mM}$ sodium pyruvate and $10 \mathrm{mM}$ HEPES) and grown to approximately $90 \%$ confluence. HUVEC-CS cells were subcultured into 12-well plates at a cell density of $4 \times 10^{4} / \mathrm{mL}$, and maintained in an incubator overnight. The cultured HUVEC-CS cells were stimulated with $1 \mu \mathrm{g} / \mathrm{mL}$ LPS for 5 hours.

\section{Animals}

The animal protocol was approved by the Animal Care and Use Committee at the University of Electronic Science and Technology of China. Sprague-Dawley (SD) rats were obtained from the Animal Center of Sichuan University (Chengdu, China). We established and characterized the SD rat atherosclerosis model in our laboratory. Rats were split into two groups: one group receiving a high fat diet with vitamin $\mathrm{D}_{3}$, and the other (control) group receiving normal feed. After more than 3 months, the animals were sacrificed. The aorta thoracalis was removed for paraffin sectioning and immunohistochemistry.

\section{Immunohistochemistry}

Staining was performed on perfusion-fixed and paraffinembedded sections. Tissue samples were fixed in cold $4 \%$ paraformaldehyde for more than 24 hours, and embedded in paraffin for sectioning. Paraffin sections from all animal groups were processed by staining with hematoxylin and eosin (H\&E). For immunohistochemistry, $4 \mu \mathrm{m}$ sections were cut, and an antigen retrieval protocol was performed. Endogenous peroxidase was eliminated using 3\% hydrogen peroxide in distilled water. The sections were incubated with blocking reagents, a polyclonal rabbit anti-rat VCAM-1 primary antibody, biotinylated anti-rabbit secondary antibody, streptavidin-horseradish peroxidase (HRP) conjugate, and 3,3'-diaminobenzidine (DAB) chromagen. The samples were examined under a microscope (TE-2000U; Nikon, Tokyo, Japan).

\section{Semi-quantitative RT-PCR}

Total RNA was isolated from cultured HUVEC-CS cells using TriZol reagent (Invitrogen, Carlsbad, CA) according to the manufacturer's instructions. Primer sequences for VCAM-1 were 5'-ATGACATGCTTGAGCCAGG-3' and 5'-GTGTCTCCTTCTTT GACACT-3' for the sense and antisense primers, respectively. ${ }^{16}$ One microgram of total RNA obtained from each sample was used for reverse transcription-PCR (RT-PCR) in a $20 \mu \mathrm{L}$ reaction mixture, and the reverse transcription was performed at $42^{\circ} \mathrm{C}$ for 90 minutes. The reverse transcriptase was inactivated at $70^{\circ} \mathrm{C}$ for $15 \mathrm{~min}$ utes. Three microliters of cDNA was amplified in a $25 \mu \mathrm{L}$ standard PCR reaction. PCR amplification was performed on a thermal cycler over 35 cycles, each cycle consisting of $94^{\circ} \mathrm{C}$ for 30 seconds, $60^{\circ} \mathrm{C}$ for 30 seconds, and $72^{\circ} \mathrm{C}$ for 1 minute. Aliquots of the $\mathrm{PCR}$ reaction products were run on $1.5 \%$ agarose gel electrophoresis and analyzed by product size, compared with a co-amplified control template.

\section{Flow cytometric determination of VCAM-I expression}

Trypsinized HUVECS-CS cells were diluted slightly in PBS and incubated with anti-human VCAM-1 monoclonal antibody at 1:5 dilution for 30 minutes. The cells were then washed with PBS, and fixed in 4\% paraformaldehyde. Flow cytometry (BD FACScanto II; BD Biosciences, Franklin 
Lakes, NJ) were used to quantify VCAM-1 expression on the surfaces of HUVECS-CS cells and primary HUVECs ( $n=3$ per condition).

\section{Shear flow simulation and microbubble adhesion}

A rectangular parallel plate perfusion chamber, designed by our laboratory, was used to examine adhesion of microbubbles to cultured HUVEC-CS monolayer under shear flow conditions. ${ }^{13}$ This system comprises a transparent polymethylmethacrylate plate, two silastic rubber gaskets, and a standard glass coverslip. The coverslip with a near $90 \%$ confluent monolayer of HUVEC-CS was mounted over the groove with the cells facing the inside, and an approximately $500 \mu \mathrm{m}$ gap was formed over the HUVEC-CS cells. The wall shear stress $\left(\tau_{w}\right)$ is related to the volumetric flow rate (Q) by $\tau_{w}=6 \mu Q / w(h)^{2}$, where $\mu$ is the fluid viscosity, $w$ is the width of the flow field, and $h$ is the height. The shear stress can be regulated through the flow rate, $Q$. The chamber was placed on the stage of an inverted microscope (TE-2000U; Nikon, Tokyo, Japan).

HUVEC-CS cells were pre-seeded on a coverslip for 48 hours. The cells were activated by LPS stimulation for 5 hours, and the cultured HUVEC-CS cells coverslip was fixed in the flow chamber for continuous shear stress exposure (6.3 or 10.4 dynes $/ \mathrm{cm}^{2}, 3$ minutes). Before perfusion the microbubble suspension was mixed with the perfusion medium. Adhesion was evaluated by counting the adhered microbubble numbers under a microscope. Six random fields (magnification $40 \times$ ) were chosen to count the microbubbles retained on the endothelial cell monolayer.

\section{Statistical analysis}

Results are expressed as arithmetic mean \pm SEM. Student's $t$-test was used to calculate the statistical significance. Differences between multiple groups were examined using ANOVA (Bonferroni $t$-test). Mean differences with a $P$ value less than 0.05 were considered to be significant. Statistical analyses were performed using SPSS software (SPSS Inc, Chicago, IL).

\section{Results}

\section{VCAM-I expression by RT-PCR,} immunohistochemistry, and flow

\section{cytometry}

VCAM-1 expression was detected by RT-PCR. It was found that VCAM-1 was unregulated after LPS stimulation, and mRNA expression presented in a dose-dependent manner as shown in Figure 2A. Furthermore, VCAM-1 immunoreactivity was observed both in normal cultured HUVEC-CS cells (control) and LPS-activated HUVEC-CS cells (Figure 2B). VCAM-1 expression in LPS-activated HUVEC-CS cells was obviously increased when compared with the control. It has been reported that other inflammatory cytokines could also upregulate the expression of some specific adhesion molecules in endothelial cells. ${ }^{17-19}$ To further confirm VCAM-1 expression quantitatively, we investigated LPS-induced VCAM-1 expression in HUVEC-CS cells and primary HUVECs. LPS-induced VCAM-1 expression in HUVEC-CS cells and primary HUVECs were both significantly higher than the control (Figure 2C). Immunohistochemical analysis also showed higher levels of VCAM-1 circumscribing the aorta thoracalis compared with controls (Figure 3), suggesting that the increased expression of VCAM-1 was partly due to increased expression of VCAM-1 on the vascular endothelial cells.
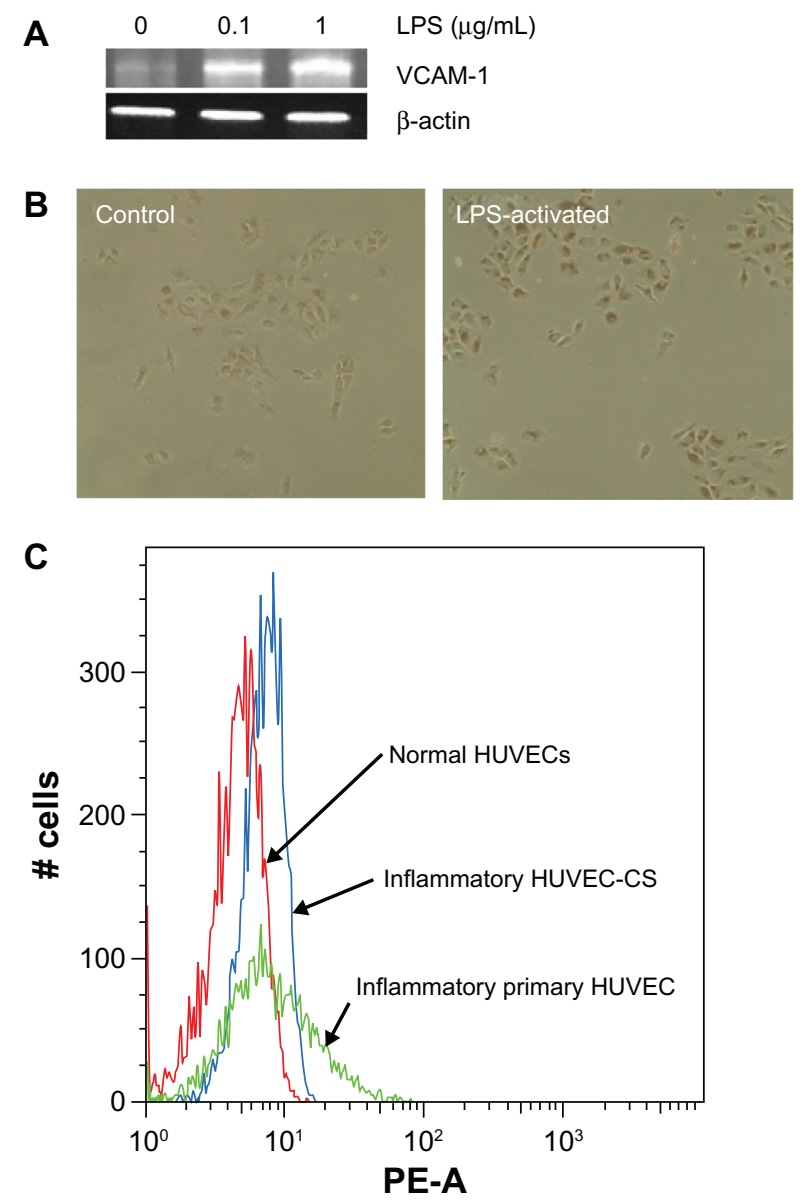

Figure 2 VCAM-I expression on HUVEC-CS cells or primary HUVECs. (A) mRNA expression of VCAM-I on HUVEC-CS cells activated by lipopolysaccharides (LPS). (B) The dark yellow shows VCAM-I expression on HUVEC-CS cells detected by immunocytochemistry. (C) Flow cytometric data using cultured HUVEC-CS cells and primary HUVECs with PE-labeled VCAM-I. The cells were either normal (control) or activated by LPS $(I \mu \mathrm{g} / \mathrm{mL})$ for 5 hours.

Abbreviations: VCAM-I, vascular cell adhesion molecule I; HUVEC-CS, human umbilical vein endothelial cells, subline; HUVEC, human umbilical vein endothelial cells; LPS, lipopolysaccharides; PE, phycoerythrin. 


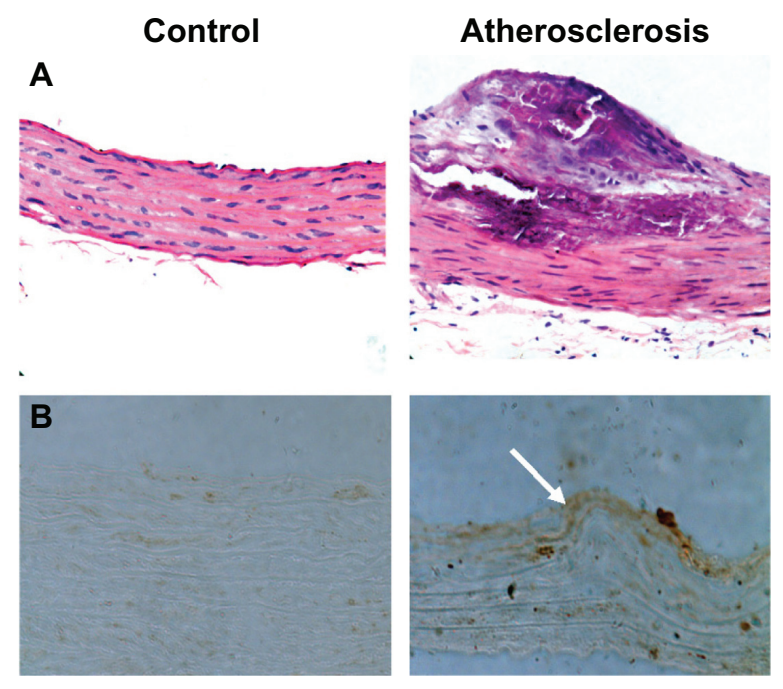

Figure 3 (A) Example of hematoxylin and eosin (H\&E) staining of aortic morphology of normal (left) and atherosclerotic SD rats (right). (B) Overexpression of VCAM-I (white arrow) on the atherosclerotic lesion detected by immunohistochemistry. Abbreviations: H\&E, hematoxylin and eosin; SD, sprague-Dawley; VCAM-I, vascular cell adhesion molecule I.

\section{Characterization of microbubbles and VCAM-I conjugation}

Figures $4 \mathrm{~A}$ and $\mathrm{B}$ show the bright field and fluorescent microscopic morphology of synthesized microbubbles, respectively. The prepared microbubbles are smooth and spherical. Our data also showed that the size of microbubbles mainly lies in the range of 2 to $5 \mu \mathrm{m}$ (Figure 4C), with a mean diameter of $\sim 3.57 \mu \mathrm{m}$. Statistical analysis showed that nearly $80 \%$ of microbubbles had diameters below $5.5 \mu \mathrm{m}$ (data not shown).

The fluorescence intensity data (relative fluorescence unit, RFU) from microbubbles incubated with various concentrations of PE-streptavidin $(0.02-1 \mu \mathrm{g} / \mathrm{mL})$ are shown in Figure 5. The conjugated PE-streptavidin densities on the microbubbles show a sigmoid distribution, and the conjugated PE-streptavidin mount reaches a saturation state when the PEstreptavidin concentration exceeds $0.6 \mu \mathrm{g} / \mathrm{mL}$. It is reasonable to assume that one streptavidin will bind one biotinylated antiVCAM-1 monoclonal antibody. Consequently, the antibody coverage percentage can be calculated through the fluorescence intensity of PE-streptavidin using a standard curve of PE-streptavidin fluorescent intensities and concentrations (data not shown). Based on this assumption, the anti-VCAM-1 antibody densities (coverage percentages) have a linear relationship to the PE-streptavidin concentration, but achieve a maximum (100\% coverage percentage) (Figure 6).

\section{Microbubble adhesion to endothelial cells under shear stress}

Microbubble adhesion was studied under several sets of flow and antigen surface density conditions. The
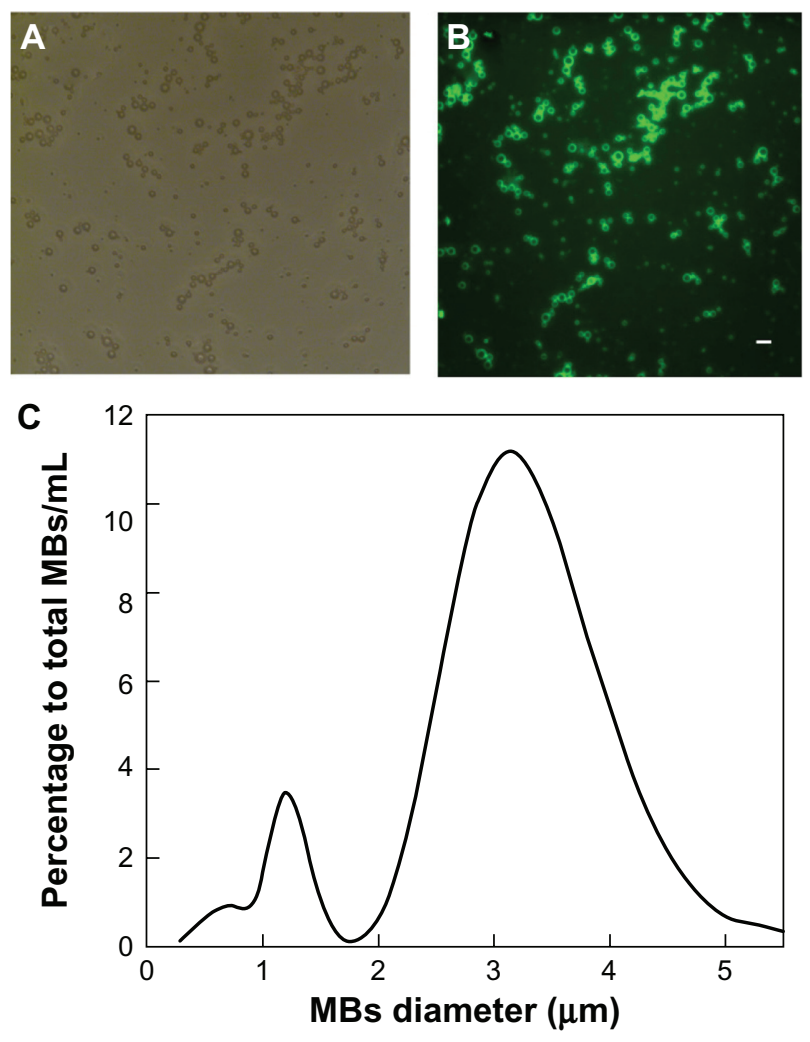

Figure 4 Characterization of synthesized microbubbles. (A) Representative bright field micrograph. (B) Representative fluorescent micrograph with DiO labeling. (C) Size distribution of microbubbles measured with a Zetasizer Nano ZS, confirming the mean diameter of the microbubbles to be $3.57 \mu \mathrm{m}$. Abbreviation: DiO, 3,3'-dioctadecyloxacarbocyanine perchlorate.

anti-VCAM-1 monoclonal antibody coverage percentages were divided into five groups of $0 \%$ (control), $22.2 \%, 32.2 \%$, $63.3 \%$, and $100 \%$. The adhesion data from coverslips of HUVEC-CS cells perfused with targeted microbubbles (conjugated anti-VCAM-1 monoclonal antibodies), control

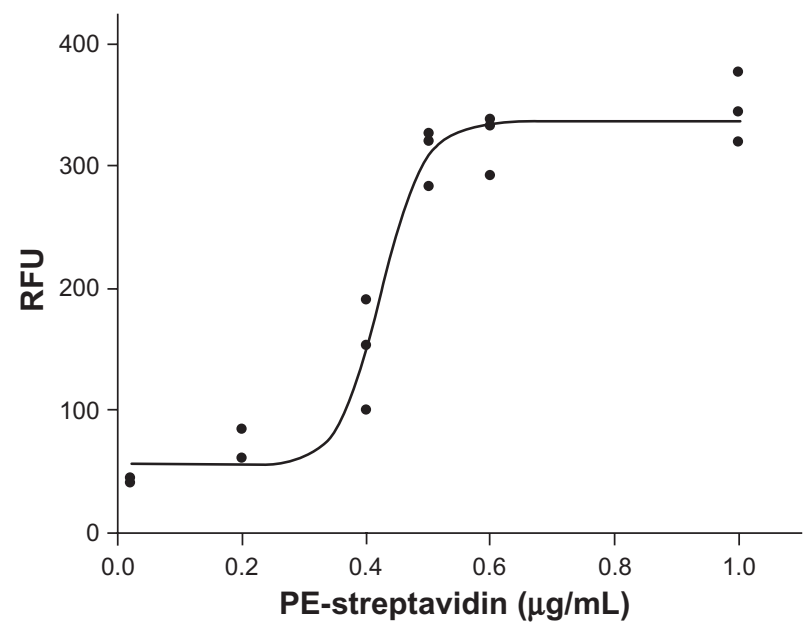

Figure $\mathbf{5}$ The fluorescence intensity of PE-streptavidin conjugated to microbubbles detected by spectrofluorophotometry. Biotinylated microbubbles were incubated with various concentrations of PE-streptavidin.

Abbreviation: PE, phycoerythrin. 


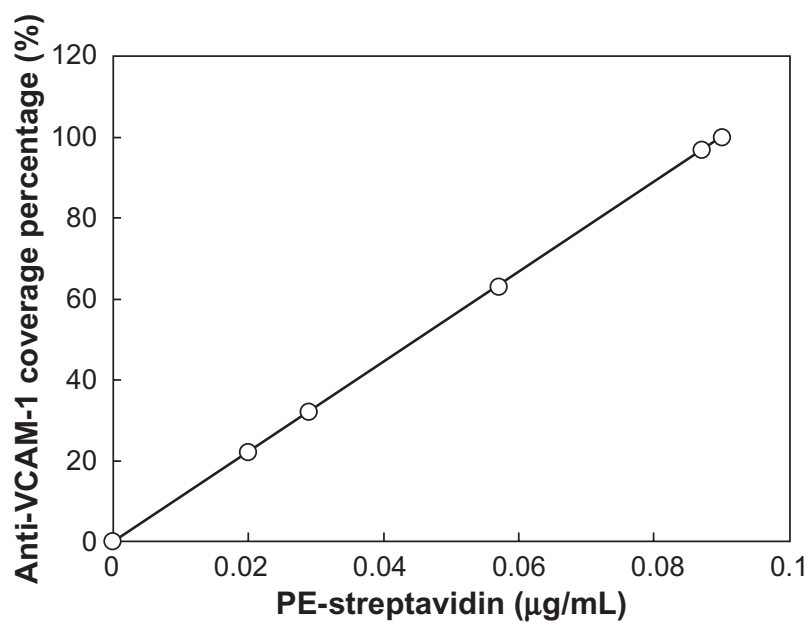

Figure 6 The calculated anti-VCAM-I coverage percentages (antibody densities) on microbubble surface under various concentrations of PE-streptavidin. The antibody coverage percentages could be evaluated through a standard curve of PE-streptavidin fluorescent intensity under various concentrations of PE-streptavidin.

Abbreviations: VCAM-I, vascular cell adhesion molecule I; PE, phycoerythrin.

microbubbles (no conjugated anit-VCAM-1) and isotype control microbubbles (conjugated nonspecific $\operatorname{IgG}$ ) are shown in Figures 7 and 8. The adhered microbubble number on the HUVEC-CS cell monolayer was quantified by microbubble counting from six random microscopic fields for each group (magnification 40×) (Figures 7B and 8B). Among the microbubbles targeted to VCAM-1, adhesion increased with increasing anti-VCAM-1 coverage percentage for LPS-activated HUVEC-CS cells. Adherence of VCAM1-targeted microbubbles was greater for LPS-activated than normal HUVEC-CS cells, and increased with increasing anti-VCAM-1 coverage percentage. There was a significant difference between the targeted microbubble groups $(22.2 \%, 32.2 \%, 63.3 \%$, and $100 \%)$ and the control microbubble group or the isotype control microbubble group.

By comparing Figures 7 and 8, the adhesion of microbubbles for each group also shows a shear-dependent relationship. Microbubble adhesion significantly decreased when the exposed shear stresses increased from 6.3 to 10.4 dynes $/ \mathrm{cm}^{2}$, which demonstrated that the interaction of microbubbles with HUVEC-CS cells depends on both bound antibody densities and flow conditions.

\section{Discussion}

Microbubbles are very effective at augmenting contrast in ultrasound images of the vasculature and enhancing visualization of the vasculature throughout the body and within organs. The advantages of using microbubbles for this purpose are that they are biocompatible, stable, small enough to travel through the entire vasculature, and highly echogenic., ${ }^{420,21}$
In this study, we developed and described a new type of bio-functionalized microbubbles, VCAM-1-targeted microbubbles, which selectively accumulate and adhere to inflamed endothelial cells in vitro under hemodynamic shear flow simulation conditions. In our previous work, we demonstrated that LPS-induced expressions of proinflammatory cytokines (IL-1 $\beta$, IL-6) and chemokines (IL-8, MCP-1) were greatly upregulated. ${ }^{22}$ LPS is a potent activator of cells in the immune and inflammatory systems, including macrophages, monocytes, and endothelial cells. In this study, we identified whether VCAM-1 expression in endothelial cells was also upregulated by LPS stimulation. The data presented in Figure 1 strongly indicates that VCAM-1 expression in LPS-activated HUVEC-CS cells is significantly higher compared to that of the control (non-LPS treated cells). LPS could activate endothelial cells through the nuclear factor- $\mathrm{\kappa B}$ pathway, and lead to expression of its targeted genes, including VCAM- $1 .{ }^{22}$

Atherosclerosis is a chronic vascular inflammation in its early stages. To verify whether VCAM-1 expression in vivo was similar to that in atherosclerogenesis, we constructed a rat model of atherosclerosis, and detected VCAM-1 expression in the endothelium. The in vivo results are consistent with the in vitro data, which suggests that VCAM-1 is an ideal target or biomarker for microbubble delivery (Figure 3). Based on these findings, we designed targeted microbubbles to conjugate the ligand of VCAM-1 (anti-VCAM-1 monoclonal antibody) in this study.

There are many factors affecting targeted microbubble adhesion to the surfaces of endothelial cells. Two important factors amongst them are the antibody coverage percentages on the microbubbles (ie, antibody surface densities) and the exposed shear stresses. Our data showed that antibody coverage percentages were positively related to microbubble adhesion number under both low $\left(6.3 \mathrm{dynes} / \mathrm{cm}^{2}\right)$ and high shear stress $\left(10.4\right.$ dynes $\left./ \mathrm{cm}^{2}\right)$ conditions. In other words, the level of microbubble adhesion increased with the antibody coverage percentages on the microbubbles. Furthermore, we found that nontargeted microbubbles (bare microbubbles) exhibited a very low adhesion to HUVEC-CS cells. Similar results were obtained for nonspecific IgG conjugated microbubbles (isotype control). The study also showed that the high adherence of anti-VCAM-1 antibody conjugated microbubbles to LPS-activated endothelial cells was specific. Our data showed that antibody coverage percentages on the microbubbles should be as high as possible in order to increase the numbers of adhered microbubbles. 


\section{A}
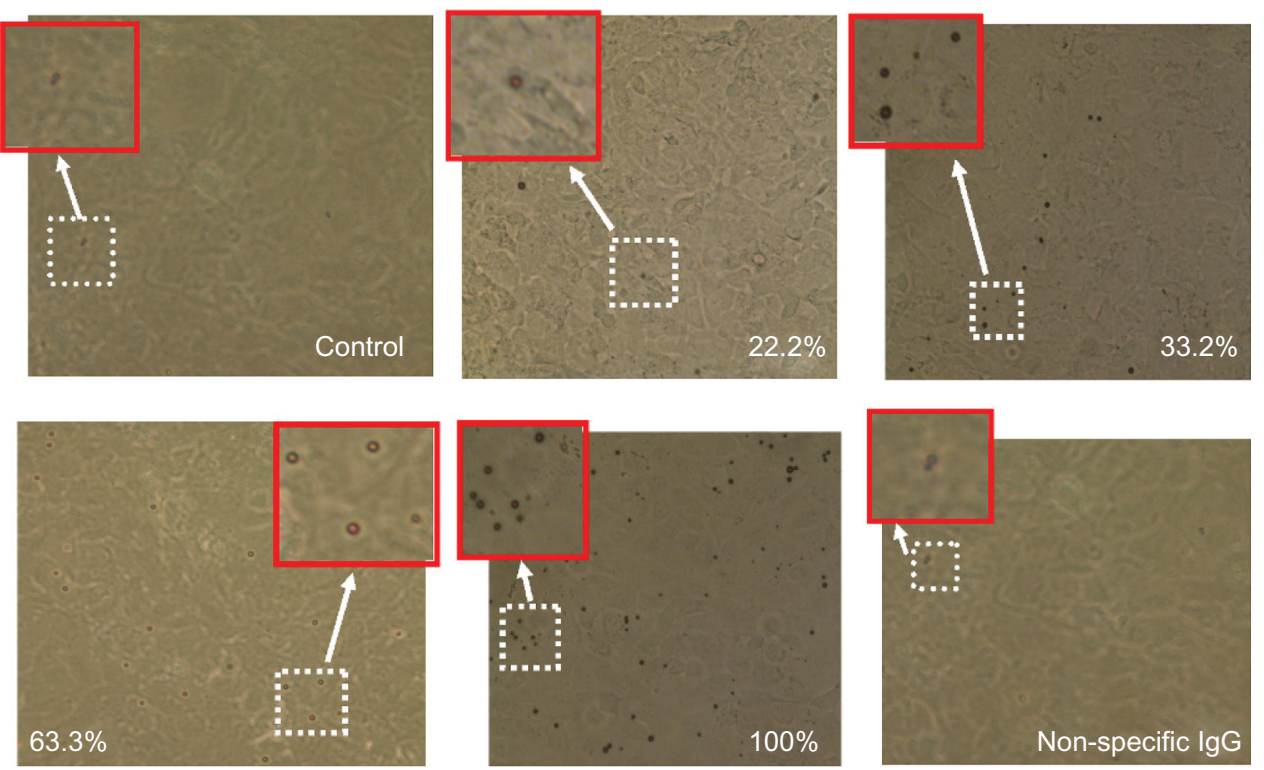

B

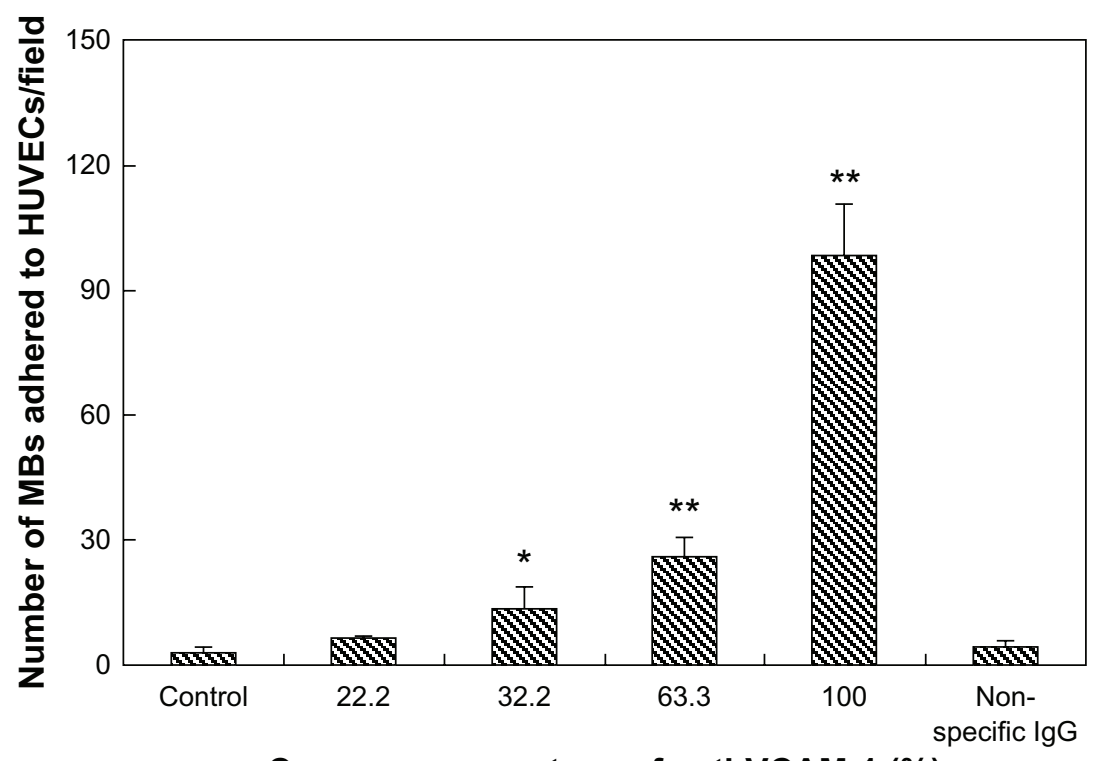

Coverage percentage of anti-VCAM-1 (\%)

Figure 7 Adhesion of control microbubbles, VCAM-I-targeted microbubbles, and isotype control lgG microbubbles under low shear stress exposure of 6.3 dynes $/ \mathrm{cm}^{2}$ for 3 minutes. (A) Representative bright field micrographs of microbubbles adhered to LPS-activated HUVEC-CS cells (magnification 40x). The insets are the high-magnification fields of the white dotted windows. (B) The adhered microbubble numbers on the HUVEC-CS cell monolayer were quantified by microbubble counting from six random microscopic fields (magnification 40x) for each group.

Notes: $* P<0.05$ vs control (non-targeted microbubbles); ${ }^{* * P}<0.01$ vs control (non-targeted microbubbles) or non-specific lgG conjugated microbubbles.

Abbreviations: VCAM-I, vascular cell adhesion molecule I; HUVEC-CS, human umbilical vein endothelial cells, subline.

Comparing the adhered number of targeted microbubbles under the two shear stress conditions, it was found that the adhered microbubble numbers decreased at the same antibody coverage percentage on the microbubble surfaces under high shear stress (Figures 7 and 8). Antibody-ligand binding may have been more easily broken under exposure to higher shear stress conditions, but adhesion was still significantly higher than in the controls. This specific adhesion could be used in clinical applications for targeted ultrasound molecular imaging and ultrasound-assisted drug/gene delivery to localized disease sites in the future. In the present study, avidin-biotin chemistry was chosen to couple the biotinylated microbubbles with biotinylated antibodies. Recently, a new protocol introduced superparamagnetic iron oxide nanoparticles (mean diameter $12 \mathrm{~nm}$ ) into the polymer shell of the microbubbles. ${ }^{23}$ This suggests that dual-targeted microbubbles with magnetic and ligand targets could be prepared, and that microbubble accumulation at disease sites could be enhanced using external magnetic fields. 
A
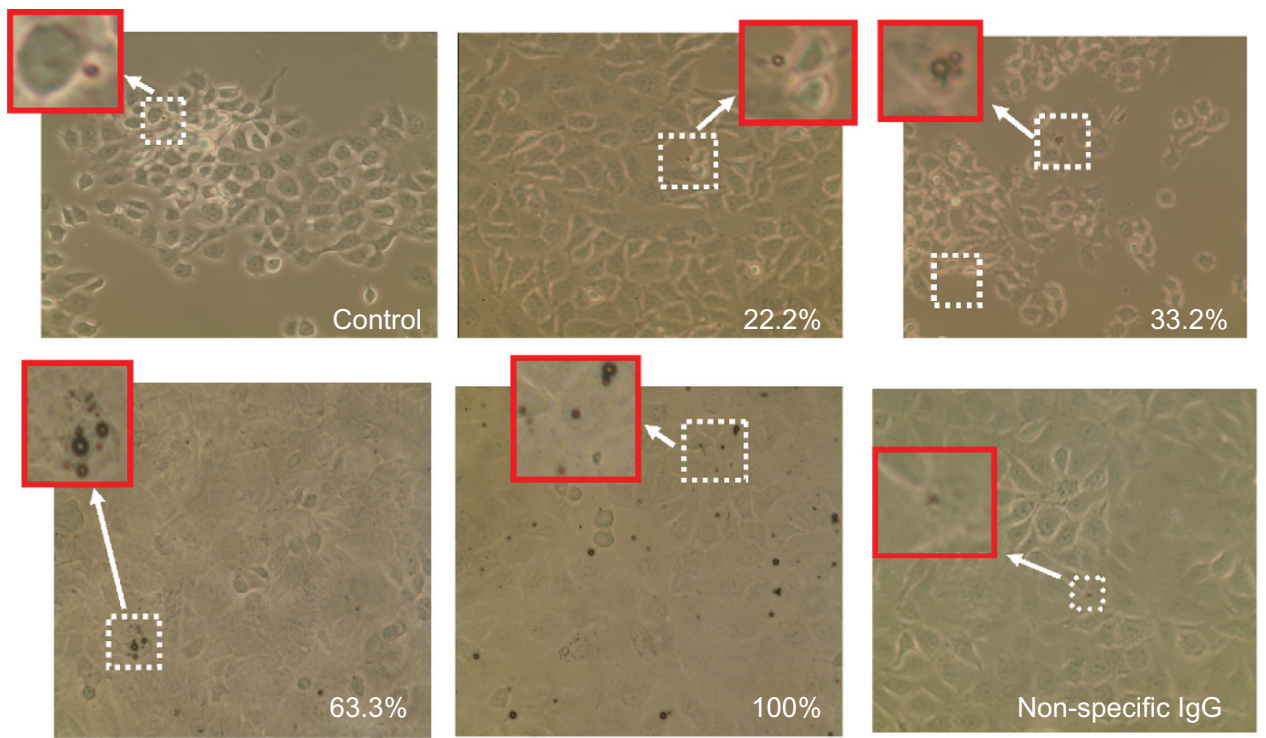

B

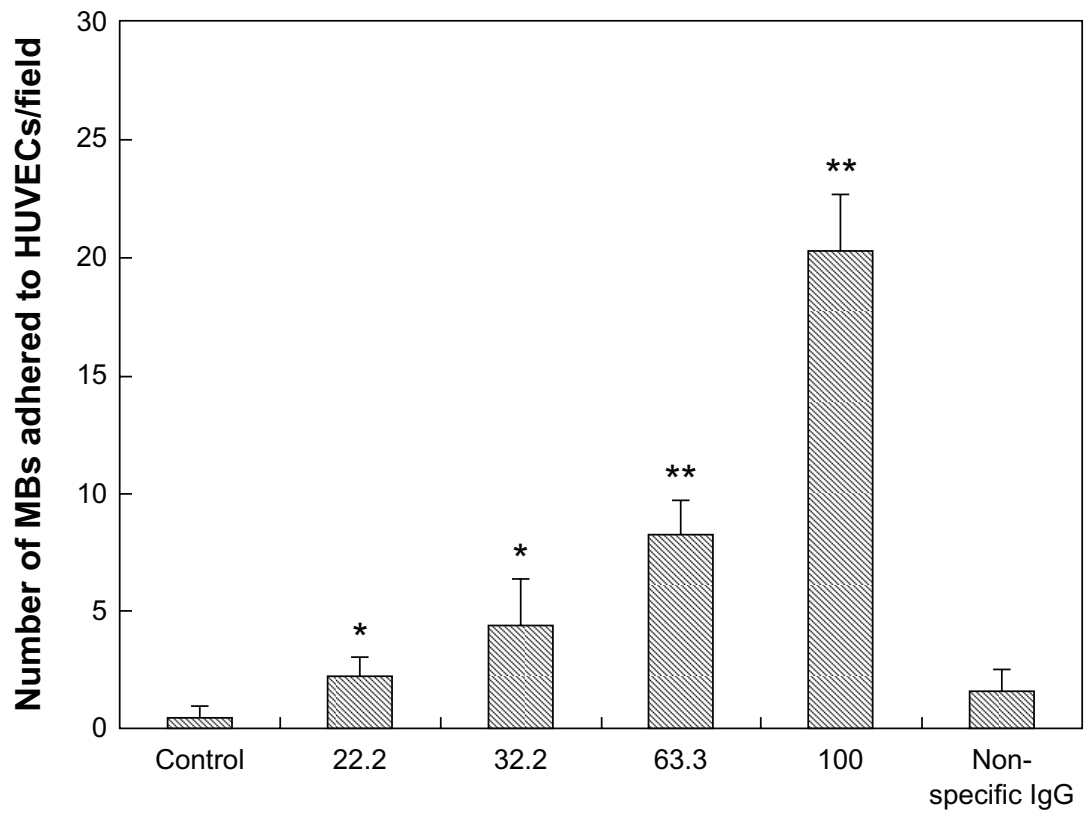

Coverage percentage of anti-VCAM-1 (\%)

Figure 8 Adhesion of control microbubbles, targeted microbubbles, and isotype control lgG microbubbles under high shear stress exposure of I0.4 dynes/cm ${ }^{2}$ for 3 minutes. (A) Representative bright field micrographs of microbubbles adhered to LPS-activated HUVEC-CS cells (magnification 40x). The insets are the high-magnification fields of the white dotted windows. (B) The adhered microbubble numbers on the HUVEC-CS cell monolayer were quantified by microbubble counting from six random microscopic fields (magnification 40X) for each group.

Notes: $* P<0.05$ versus control (non-targeted microbubbles); $* * P<0.0$ I versus control (non-targeted microbubbles) or non-specific lgG conjugated microbubbles.

Abbreviations: LPS, lipopolysaccharide; HUVEC-CS, human umbilical vein endothelial cells, subline.

\section{Conclusion}

In summary, we have engineered a novel type of biofunctionalized ultrasound microbubbles (VCAM-1-targeted microbubbles) with an average diameter of $3.57 \mu \mathrm{m}$ using multi-step biotin-avidin bridging chemistry methods. The targeted microbubbles can specifically adhere to inflammatory endothelial cells activated by LPS, and their adherence depends on the antibody coverage percentages on the microbubbles and hemodynamic shear flow conditions.
The bio-functionalized microbubbles have promising applications in targeted ultrasound molecular imaging and targeted ultrasound-assisted drug delivery in the future.

\section{Acknowledgments}

We would like to acknowledge financial support from the National Natural Science Foundation of China (30700151, 81071257), New Century Excellent Talents Program in Chinese Universities (NCET-09-0263), Sichuan Youth 
Science and Technology Foundation of China (2010 JQ0004), Fundamental Research Funds for Central Universities (23801135, 23801131), and Visiting Scholar Foundation of Key Lab of Biorheological Science and Technology in Chongqing University, Ministry of Education, China.

\section{Disclosure}

The authors report no conflicts of interest in this work.

\section{References}

1. Lindner JR. Microbubbles in medical imaging: current applications and future directions. Nat Rev Drug Discov. 2004;3(6):527-532.

2. Sirsi S, Borden M. Microbubble compositions, properties and biomedical applications. Bubble Sci Eng Technol. 2009;1(1-2):3-17.

3. Takalkar AM, Klibanov AL, Rychak JJ, Lindner JR, Ley K. Binding and detachment dynamics of microbubbles targeted to P-selectin under controlled shear flow. J Control Release. 2004;96(3):473-482.

4. Weller GE, Villanueva FS, Klibanov AL, Wagner WR. Modulating targeted adhesion of an ultrasound contrast agent to dysfunctional endothelium. Ann Biomed Eng. 2002;30(8):1012-1019.

5. Liu Y, Miyoshi H, Nakamura M. Encapsulated ultrasound microbubbles: therapeutic application in drug/gene delivery. $J$ Control Release. 2006;114(1):89-99.

6. Liu Y, Uno H, Takatsuki H, Hirano M, Sakanishi A. Interrelation between HeLa-S3 cell transfection and hemolysis in red blood cell suspension using pulsed ultrasound of various duty cycles. Eur Biophys $J$. 2005;34(2):163-169.

7. Liu Y, Yang H, Sakanishi A. Ultrasound: mechanical gene transfer into plant cells by sonoporation. Biotechnol Adv. 2006;24(1):1-16.

8. Sakhalkar HS, Hanes J, Fu J, et al. Enhanced adhesion of ligandconjugated biodegradable particles to colitic venules. FASEB J. 2005; 19(7):792-794.

9. Allen AR, McHale J, Smith J, et al. Endothelial expression of VCAM-1 in experimental crescentic nephritis and effect of antibodies to very late antigen-4 or VCAM-1 on glomerular injury. J Immunol. 1999;162(9):5519-5527.

10. Fruebis J, Silvestre M, Shelton D, Napoli C, Palinski W. Inhibition of VCAM-1 expression in the arterial wall is shared by structurally different antioxidants that reduce early atherosclerosis in NZW rabbits. J Lipid Res. 1999;40(11):1958-1966.

11. Lei X, Lawrence MB, Dong C. Influence of cell deformation on leukocyte rolling adhesion in shear flow. J Biomech Eng. 1999;121(6): 636-643.
12. Liang S, Dong C. Integrin VLA-4 enhances sialyl-Lewisx/a-negative melanoma adhesion to and extravasation through the endothelium under low flow conditions. Am J Physiol Cell Physiol. 2008; 295(3):C701-C707.

13. Liu Y, Zhao F, Gu W, et al. The roles of platelet GPIIb/IIIa and alphavbeta 3 integrins during HeLa cells adhesion, migration, and invasion to monolayer endothelium under static and dynamic shear flow. J Biomed Biotechnol. 2009;2009:829243.

14. Kaufmann BA, Lewis C, Xie A, Mirza-Mohd A, Lindner JR Detection of recent myocardial ischaemia by molecular imaging of P-selectin with targeted contrast echocardiography. Eur Heart $J$. 2007;28(16):2011-2017.

15. Gifford SM, Grummer MA, Pierre SA, et al. Functional characterization of HUVEC-CS: Ca2+ signaling, ERK 1/2 activation, mitogenesis and vasodilator production. $J$ Endocrinol. 2004;182(3):485-499.

16. Kaplanski G, Marin V, Fabrigoule M, et al. Thrombin-activated human endothelial cells support monocyte adhesion in vitro following expression of intercellular adhesion molecule-1 (ICAM-1; CD54) and vascular cell adhesion molecule-1 (VCAM-1; CD106). Blood. 1998;92(4):1259-1267.

17. Sawa Y, Ueki T, Hata M, et al. LPS-induced IL-6, IL-8, VCAM-1, and ICAM-1 expression in human lymphatic endothelium. $J$ Histochem Cytochem. 2008;56(2):97-109.

18. Shi Q, Wang J, Wang XL, et al. Comparative analysis of vascular endothelial cell activation by TNF-alpha and LPS in humans and baboons. Cell Biochem Biophys. 2004;40(3):289-303.

19. Zeuke S, Ulmer AJ, Kusumoto S, Katus HA, Heine H. TLR4-mediated inflammatory activation of human coronary artery endothelial cells by LPS. Cardiovasc Res. 2002;56(1):126-134.

20. Rychak JJ, Lindner JR, Ley K, Klibanov AL. Deformable gasfilled microbubbles targeted to P-selectin. J Control Release. 2006; 114(3):288-299.

21. Villanueva FS, Jankowski RJ, Klibanov S, et al. Microbubbles targeted to intercellular adhesion molecule-1 bind to activated coronary artery endothelial cells. Circulation. 1998;98(1):1-5.

22. Meng Q, Liu Y, Lou C, et al. Emodin suppresses LPS-induced proinflammatory responses and NF- $\mathrm{KB}$ activation by disruption of lipid rafts in CD14 negative endothelial cells. $\mathrm{Br} J$ Pharmacol. 2010;161(7):1628-1644.

23. Yang F, Li Y, Chen Z, Zhang Y, Wu J, Gu N. Superparamagnetic iron oxide nanoparticle-embedded encapsulated microbubbles as dual contrast agents of magnetic resonance and ultrasound imaging. Biomaterials. 2009;30(23-24):3882-3890.
International Journal of Nanomedicine

\section{Publish your work in this journal}

The International Journal of Nanomedicine is an international, peerreviewed journal focusing on the application of nanotechnology in diagnostics, therapeutics, and drug delivery systems throughou the biomedical field. This journal is indexed on PubMed Central, MedLine, CAS, SciSearch ${ }^{\circledR}$, Current Contents ${ }^{\circledR} /$ Clinical Medicine,

\section{Dovepress}

Journal Citation Reports/Science Edition, EMBase, Scopus and the Elsevier Bibliographic databases. The manuscript management system is completely online and includes a very quick and fair peer-review system, which is all easy to use. Visit http://www.dovepress.com/ testimonials.php to read real quotes from published authors. 